The Kronecker product and some of its physical applications

This content has been downloaded from IOPscience. Please scroll down to see the full text.

2016 Eur. J. Phys. 37065403

(http://iopscience.iop.org/0143-0807/37/6/065403)

View the table of contents for this issue, or go to the journal homepage for more

Download details:

IP Address: 200.130.19.129

This content was downloaded on 10/09/2016 at 14:37

Please note that terms and conditions apply.

You may also be interested in:

Quantum Mechanics: Angular momentum

M Saleem

Discrete Quantum Mechanics: Quantum angular momentum

H T Williams

Quantum Information in Gravitational Fields: Relativistic quantum fields

M Lanzagorta

An Introduction to the Formalism of Quantum Information with Continuous Variables:

Quantum-mechanical description of physical systems

C Navarrete-Benlloch

Magnetic Excitations and Geometric Confinement: Magnetism theory: spin models

G M Wysin

Quantum phase transitions in the collective degrees of freedom: nuclei and other many-body systems

Pavel Cejnar and Pavel Stránský

Minimum time control of a pair of two-level quantum systems with opposite drifts

Raffaele Romano and Domenico D'Alessandro 


\title{
The Kronecker product and some of its physical applications
}

\author{
Francisco M Fernández \\ División Química Teórica, INIFTA (CONICET, UNLP), Blvd. 113 S/N, Sucursal 4, \\ Casilla de Correo 16, 1900 La Plata, Argentina \\ E-mail: fernande@quimica.unlp.edu.ar
}

Received 4 July 2016, revised 31 July 2016

Accepted for publication 10 August 2016

Published 7 September 2016

\begin{abstract}
In a typical introductory course on quantum mechanics the student has to face two quite different operator products. One of them leads to, for example, commutators and the uncertainty principle, and the other appears when one goes from a one-particle system to a multi-particle one. The latter gives rise to the direct or tensor product of operators and vector spaces, and to the Kronecker product of the matrix representations of the operators. We illustrate the practical utility of the Kronecker product by means of two spin systems commonly discussed in introductory courses on quantum mechanics and also by means of a simple model for the interpretation of the NMR signal produced by two interacting protons.
\end{abstract}

Keywords: Kronecker product, spin systems, NMR spectrum, fine structure, operator product, matrix product

\section{Introduction}

In quantum mechanics every measurable physical quantity is described by an Hermitian operator defined on the state space of the system [1,2]. Thus, the student of an introductory course on quantum mechanics should become familiar with concepts like the sum, product, commutator, etc, of linear operators. In particular, the student should deal with at least two operator products: one is given by the composition of two operators defined on the same vector space and the other is the direct or tensor product of operators. The former product yields an operator defined on the common vector space of the factor operators, while the latter leads to an operator defined on a different vector space: the direct or tensor product of the vector spaces of the factor operators. These products are commonly discussed in most textbooks on quantum mechanics [1, 2]. However, the special symbol for the tensor product is typically omitted in many textbooks (in particular, it rarely appears in quantum chemistry ones [3]). Although this omission is clearly indicated in most good textbooks [2] it may 
nevertheless lead to some misunderstanding when one starts the discussion of composite systems. In particular expressions of the form $\hat{H}=\hat{H}_{1}+\hat{H}_{2}$, where $\hat{H}_{i}$ is the Hamiltonian operator for the system $i$, are misleading because they appear to suggest that we are adding two operators defined on different vector spaces (the state space of system 1 and the state space of system 2). The origin of this confusion can be traced back to the abandonment of the symbol for the tensor product just mentioned.

In some specialized scientific textbooks the tensor product is introduced rigorously, discussed in detail and applied systematically to problems that involve composite systems. Illustrative examples are those aimed at the study of quantum computation and information [4-6].

The purpose of this paper is to show, in a simple, pedagogical way, why it is convenient to keep the notation of the tensor product explicitly in some particular cases (one can abandon it later if desired) to avoid the misunderstanding mentioned above. In addition, we will also show that the explicit application of the tensor product of operators and the resulting Kronecker product of matrices is quite useful for solving quantum-mechanical problems in a simple, straightforward way. In section 2 we outline the operator products just mentioned. Section 3 shows the practical application of the Kronecker product of matrices to a twoelectron spin system. In section 4 this treatment is extended to the case of three electrons to illustrate that the application of the technique to a larger number of particles is straightforward. In section 5 we briefly discuss a model of two interacting spins that simulates the problem posed by two protons in a magnetic field that give rise to an NMR signal with fine structure [7]. Finally, in section 6 we discuss the main results of the paper and draw conclusions.

\section{Product of operators}

In this section we outline some well known concepts commonly discussed, with varying degrees of rigour, in most textbooks on quantum mechanics [1, 2] and quantum chemistry [3]. For simplicity we consider a linear operator $\hat{A}$ defined on an $N$-dimensional vector space $\bar{S}_{f}$ and let $S_{f}=\left\{f_{1}, f_{2}, \ldots, f_{N}\right\}$ be an orthonormal basis set for $\bar{S}_{f}$. We will resort to the standard quantum-mechanical notation $\left\langle f_{i} \mid f_{j}\right\rangle=\delta_{i j}$ for the scalar product in $\bar{S}_{f}$. The elements $A_{i j}$ of the matrix representation $\mathbf{A}$ of $\hat{A}$ in the basis $S_{f}$ are given by

$$
A f_{j}=\sum_{i=1}^{N} A_{i j} f_{i}
$$

If $\hat{B}$ is another linear operator defined on $\bar{S}_{f}$ we can define the product $\hat{A} \hat{B}$ in the following way: $(\hat{A} \hat{B}) f=\hat{A}(\hat{B} f)$ for every $f \in \bar{S}_{f}$. This product is similar to the function composition $(F \circ G)(x)=F(G(x))$ but we simply write $\hat{A} \hat{B}$ (as is custommary in quantum mechanics) instead of $\hat{A} \circ \hat{B}$. If $\mathbf{B}=\left(B_{i j}\right)$ is the matrix representation of $\hat{B}$ in the basis $S_{f}$ then

$$
\hat{A} \hat{B} f_{j}=\hat{A} \sum_{m=1}^{N} B_{m j} f_{m}=\sum_{m=1}^{N} B_{m j} \sum_{i=1}^{N} A_{i m} f_{i}=\sum_{i=1}^{N}\left(\sum_{m=1}^{N} A_{i m} B_{m j}\right) f_{i},
$$

shows that $\mathbf{A B}$ is the matrix representation of $\hat{A} \hat{B}$.

The sum $\hat{A}+\hat{B}$ of two linear operators is defined as $(\hat{A}+\hat{B}) f=\hat{A} f+\hat{B} f$ for every $f \in \bar{S}_{f}$. It therefore follows from the arguments given above that the matrix representation of $\hat{A}+\hat{B}$ is simply $\mathbf{A}+\mathbf{B}$. 
The operator product outlined above is not the only one that we commonly use in quantum mechanics. Suppose that the linear operator $\hat{B}$ is defined on a different vector space $\bar{S}_{g}$ with orthonormal basis $S_{g}=\left\{g_{1}, g_{2}, \ldots, g_{M}\right\}$. In order to treat the composite system given by two operators defined on different vector spaces we define the vector space $\bar{S}_{f g}=\bar{S}_{f} \otimes \bar{S}_{g}$ and the orthonormal basis $S_{f g}=\left\{u_{i j}=f_{i} \otimes g_{j}, i=1,2, \ldots, N, j=1,2, \ldots, M\right\}$, where $\otimes$ is commonly known as the direct or tensor product [1, 2, 4-6]. Any vector $f$ in $\bar{S}_{f g}$ can be written as a linear combination of the elements of this basis set

$$
f=\sum_{i=1}^{N} \sum_{j=1}^{M} c_{i j} u_{i j}
$$

The scalar product in this vector space is given by

$$
\left\langle u_{i j} \mid u_{m n}\right\rangle=\left\langle f_{i} \mid f_{m}\right\rangle\left\langle g_{j} \mid g_{n}\right\rangle=\delta_{i m} \delta_{j n} .
$$

In this case we define a different operator product $\hat{A} \otimes \hat{B}$ in the following way

$$
(\hat{A} \otimes \hat{B}) u_{i j}=\left(\hat{A} f_{i}\right) \otimes\left(\hat{B} g_{j}\right) .
$$

Therefore

$$
(\hat{A} \otimes \hat{B}) u_{i j}=\sum_{n=1}^{N} \sum_{m=1}^{M} A_{n i} B_{m j} f_{n} \otimes g_{m}=\sum_{n=1}^{N} \sum_{m=1}^{M} A_{n i} B_{m j} u_{n m},
$$

shows that the matrix representation of $\hat{A} \otimes \hat{B}$ is given by the $N M \times N M$ matrix with elements $A_{n i} B_{m j}, n, i=1,2, \ldots N, m, j=1,2, \ldots, M$. The resulting larger matrix is known as the Kronecker product of the matrices $\mathbf{A}$ and $\mathbf{B}$ and is denoted by $\mathbf{A} \otimes \mathbf{B}$.

In order to construct the Kronecker product $\mathbf{A} \otimes \mathbf{B}$ we just follow a simple and straighforward rule: substitute $A_{i j} \mathbf{B}$ for every element $A_{i j}$ of $\mathbf{A}$; for example:

$$
\left(\begin{array}{ll}
A_{11} & A_{12} \\
A_{21} & A_{22}
\end{array}\right) \otimes\left(\begin{array}{ll}
B_{11} & B_{12} \\
B_{21} & B_{22}
\end{array}\right)=\left(\begin{array}{llll}
A_{11} B_{11} & A_{11} B_{12} & A_{12} B_{11} & A_{12} B_{12} \\
A_{11} B_{21} & A_{11} B_{22} & A_{12} B_{21} & A_{12} B_{22} \\
A_{21} B_{11} & A_{21} B_{12} & A_{22} B_{11} & A_{22} B_{12} \\
A_{21} B_{21} & A_{21} B_{22} & A_{22} B_{21} & A_{22} B_{22}
\end{array}\right) .
$$

It is not difficult to verify that in general, $\mathbf{A} \otimes \mathbf{B} \neq \mathbf{B} \otimes \mathbf{A}$. The Kronecker product also applies to rectangular matrices. For example, if $\mathbf{A}$ and $\mathbf{B}$ are $M \times N$ and $P \times Q$ matrices, respectively, then $\mathbf{A} \otimes \mathbf{B}$ is a $M P \times N Q$ matrix that one obtains by means of the simple rule just mentioned. Throughout this paper we will apply it to some illustrative examples and will refer to it as the Kronecker-product rule. It satisfies the following properties:

$$
\begin{aligned}
\mathbf{A} \otimes(\mathbf{B}+\mathbf{C}) & =\mathbf{A} \otimes \mathbf{B}+\mathbf{A} \otimes \mathbf{C}, \\
(\mathbf{A}+\mathbf{B}) \otimes \mathbf{C} & =\mathbf{A} \otimes C+\mathbf{B} \otimes \mathbf{C}, \\
(k \mathbf{A}) \otimes \mathbf{B} & =\mathbf{A} \otimes(k \mathbf{B})=k(\mathbf{A} \otimes \mathbf{B}), \\
(\mathbf{A} \otimes \mathbf{B}) \otimes \mathbf{C} & =\mathbf{A} \otimes(\mathbf{B} \otimes \mathbf{C}),
\end{aligned}
$$

where $k$ is a complex number. We will not prove these equalities here but will use them in the applications discussed in the following sections.

\section{Spin operators and matrices for one and two electrons}

In nonrelativistic quantum mechanics the spin of the particles (electrons, nuclei, etc) is introduced as an additional intrinsic angular momentum with components $\left(\hat{s}_{x}, \hat{s}_{y}, \hat{s}_{z}\right)$ (or 
$\left(\hat{I}_{x}, \hat{I}_{y}, \hat{I}_{z}\right)$ for nuclei) [1-3]. Since the components of the spin angular momentum do not commute it is custommary to describe the properties of the system by means of the eigenvectors (spin states) of $\hat{s}^{2}=\hat{s}_{x}^{2}+\hat{s}_{y}^{2}+\hat{s}_{z}^{2}$ and $\hat{s}_{z}$. The spin states of a particle are commonly denoted by $\left|s, m_{s}\right\rangle$ (or $\left.\left|I, m_{I}\right\rangle\right)$ and in the particular case of the electron $(s=1 / 2)$ they are also written [3]

$$
\alpha=|1 / 2,1 / 2\rangle, \beta=|1 / 2,-1 / 2\rangle,
$$

which satisfy

$$
s_{z} \alpha=\frac{1}{2} \alpha, s_{z} \beta=-\frac{1}{2} \beta, s^{2} \alpha=\frac{3}{4} \alpha, s^{2} \beta=\frac{3}{4} \beta .
$$

In the case of two electrons the spin components are commonly written in the form

$$
\hat{S}_{q}=\hat{s}_{q}(1)+\hat{s}_{q}(2)
$$

where $q=x, y, z$ and $\hat{s}_{q}(i)$ refers to electron $i$. The possible spin states of the two-electron system are linear combinations of the four new vectors

$$
\alpha(1) \alpha(2), \alpha(1) \beta(2), \beta(1) \alpha(2), \beta(1) \beta(2),
$$

where it is understood that

$$
\hat{S}_{q} \alpha(1) \alpha(2)=\left[\hat{s}_{q}(1) \alpha(1)\right] \alpha(2)+\alpha(1) \hat{s}_{q}(2) \alpha(2) .
$$

The two-electron spin vectors (12) are eigenvectors of $\hat{S}_{z}$ but they are not eigenvectors of

$$
\hat{S}^{2}=\hat{S}_{x}^{2}+\hat{S}_{y}^{2}+\hat{S}_{z}^{2}
$$

Therefore, we should find suitable linear combinations of the vectors (12) that satisfy the latter requirement. One can do it easily by means of straightforward inspection or, more systematically, by means of suitable ladder operators [3]. Although this notation is widely used and does not give rise to any serious difficulty we think that it is more instructive to use the direct or tensor product outlined above and write

$$
\left|1 / 2, m_{s_{1}}, 1 / 2, m_{s_{2}}\right\rangle=\left|1 / 2, m_{s_{1}}\right\rangle \otimes\left|1 / 2, m_{s_{2}}\right\rangle
$$

or, for example, $\alpha(1) \otimes \alpha(2)$ instead of $\alpha(1) \alpha(2)$. Within this more explicit notation we should write

$$
\hat{S}_{q}=\hat{s}_{q}(1) \otimes \hat{1}(2)+\hat{1}(1) \otimes \hat{s}_{q}(2),
$$

where $\hat{1}(i)$ is the identity operator defined on the spin vector space of the electron $i$. The application of this operator to, for example, $\alpha(1) \otimes \alpha(2)$ leads to equation (13) in a straightforward an possibly clearer way. A criticism of equation (11) is that it appears to suggest the sum of two operators defined on two different vector spaces (one for electron 1 and the other for electron 2). On the other hand, equation (16) explicitly indicates the sum of two operators defined on the common spin state space of the two-electron system.

What we have outlined above is not merely a change of notation to describe the same treatment of a two-electron system in a more elegant way. It enables us to obtain useful results in a straightforward and systematic way. To appreciate this statement more clearly we consider the well known dimensionless spin matrices (in units of $\hbar$ ) 


$$
\begin{aligned}
& \mathbf{s}_{x}=\frac{1}{2}\left(\begin{array}{ll}
0 & 1 \\
1 & 0
\end{array}\right), \mathbf{s}_{y}=\frac{1}{2}\left(\begin{array}{cc}
0 & -i \\
i & 0
\end{array}\right), \mathbf{s}_{z}=\frac{1}{2}\left(\begin{array}{ll}
1 & 0 \\
0 & -1
\end{array}\right), \quad \mathbf{I}=\left(\begin{array}{ll}
1 & 0 \\
0 & 1
\end{array}\right), \\
& \mathbf{s}^{2}=\mathbf{s}_{x}^{2}+\mathbf{s}_{y}^{2}+\mathbf{s}_{z}^{2}=\frac{3}{4}\left(\begin{array}{ll}
1 & 0 \\
0 & 1
\end{array}\right),
\end{aligned}
$$

to which we added the $2 \times 2$ identity matrix $\mathbf{I}$ for completeness (note that $\mathbf{s}_{q}^{2}=\frac{1}{4} \mathbf{I}$ ). The corresponding eigenvectors of $\mathbf{s}^{2}$ and $\mathbf{s}_{z}$ are

$$
\boldsymbol{\alpha}=\left(\begin{array}{l}
1 \\
0
\end{array}\right), \boldsymbol{\beta}=\left(\begin{array}{l}
0 \\
1
\end{array}\right)
$$

If we apply the Kronecker-product rule discussed at the end of section 2 we easily obtain the $4 \times 4$ matrices $\mathbf{S}_{q}$ for the two-electron system

$$
\begin{aligned}
& \mathbf{S}_{x}=\mathbf{s}_{x} \otimes \mathbf{I}+\mathbf{I} \otimes \mathbf{s}_{x}=\frac{1}{2}\left(\begin{array}{llll}
0 & 1 & 1 & 0 \\
1 & 0 & 0 & 1 \\
1 & 0 & 0 & 1 \\
0 & 1 & 1 & 0
\end{array}\right), \\
& \mathbf{S}_{y}=\mathbf{s}_{y} \otimes \mathbf{I}+\mathbf{I} \otimes \mathbf{s}_{y}=\frac{1}{2}\left(\begin{array}{llll}
0 & -i & -i & 0 \\
i & 0 & 0 & -i \\
i & 0 & 0 & -i \\
0 & i & i & 0
\end{array}\right), \\
& \mathbf{S}_{z}=\mathbf{s}_{z} \otimes \mathbf{I}+\mathbf{I} \otimes \mathbf{S}_{z}=\left(\begin{array}{llll}
1 & 0 & 0 & 0 \\
0 & 0 & 0 & 0 \\
0 & 0 & 0 & 0 \\
0 & 0 & 0 & -1
\end{array}\right), \\
& \mathbf{S}^{2}=\mathbf{S}_{x}^{2}+\mathbf{S}_{y}^{2}+\mathbf{S}_{z}^{2}=\left(\begin{array}{llll}
2 & 0 & 0 & 0 \\
0 & 1 & 1 & 0 \\
0 & 1 & 1 & 0 \\
0 & 0 & 0 & 2
\end{array}\right) .
\end{aligned}
$$

Note that $\mathbf{S}_{z}$ is diagonal because the direct products

$$
\boldsymbol{\alpha} \otimes \boldsymbol{\alpha}=\left(\begin{array}{l}
1 \\
0 \\
0 \\
0
\end{array}\right), \quad \boldsymbol{\alpha} \otimes \boldsymbol{\beta}=\left(\begin{array}{l}
0 \\
1 \\
0 \\
0
\end{array}\right), \boldsymbol{\beta} \otimes \boldsymbol{\alpha}=\left(\begin{array}{l}
0 \\
0 \\
1 \\
0
\end{array}\right), \quad \boldsymbol{\beta} \otimes \boldsymbol{\beta}=\left(\begin{array}{l}
0 \\
0 \\
0 \\
1
\end{array}\right)
$$

are its eigenvectors; but they are not eigenvectors of $\mathbf{S}^{2}$ which, for this reason, is not diagonal. From the diagonal elements of $\mathbf{S}_{z}$ we conclude that its eigenvalues are $M_{S}=-1,0,0,1$ (note that there are two eigenvectors, $\boldsymbol{\alpha} \otimes \boldsymbol{\beta}$ and $\boldsymbol{\beta} \otimes \boldsymbol{\alpha}$, with the same eigenvalue $M_{S}=0$ ).

It is not difficult to obtain the eigenvalues and eigenvectors of the matrix $\mathbf{S}^{2}$. Its characteristic polynomial $\left|\mathbf{S}^{2}-\lambda \mathbf{I}\right|=\lambda(\lambda-2)^{3}=0$, where $\mathbf{I}$ is the $4 \times 4$ identity matrix, shows that there is one eigenvector with eigenvalue $\lambda=0$ and three eigenvectors with eigenvalue $\lambda=2$. On solving the equation $S(S+1)=\lambda$ we realize that the posible spin values are $S=0$ (singlet state) and $S=1$ (triplet state). This result is consistent with the well known formula that yields the possible spins $S$ of a system of two particles with spins $s_{1}$ and $s_{2}$ as $S=\left|s_{1}-s_{2}\right|,\left|s_{1}-s_{2}\right|+1, \ldots, s_{1}+s_{2}$. In the present case, $s_{1}=s_{2}=1 / 2$ leads to $S=0,1$. Besides, the eigenvalue $\lambda=2(S=1)$ appears three times because $M_{S}=-1,0,1$ whereas $\lambda=0(S=0)$ is related to only one eigenvector with $M_{S}=0$. 
It is not difficult to verify that the eigenvectors of $\mathbf{S}^{2}$ are

$$
\begin{aligned}
& \left|S=0, M_{S}=0\right\rangle=\frac{1}{\sqrt{2}}\left(\begin{array}{r}
0 \\
1 \\
-1 \\
0
\end{array}\right)=\frac{1}{\sqrt{2}}[\boldsymbol{\alpha} \otimes \boldsymbol{\beta}-\boldsymbol{\beta} \otimes \boldsymbol{\alpha}], \\
& \left|S=1, M_{S}=1\right\rangle=\left(\begin{array}{l}
1 \\
0 \\
0 \\
0
\end{array}\right)=\boldsymbol{\alpha} \otimes \boldsymbol{\alpha} \\
& \left|S=1, M_{S}=0\right\rangle=\frac{1}{\sqrt{2}}\left(\begin{array}{l}
0 \\
1 \\
1 \\
0
\end{array}\right)=\frac{1}{\sqrt{2}}[\boldsymbol{\alpha} \otimes \boldsymbol{\beta}+\boldsymbol{\beta} \otimes \boldsymbol{\alpha}], \\
& \left|S=1, M_{S}=-1\right\rangle=\left(\begin{array}{l}
0 \\
0 \\
0 \\
1
\end{array}\right)=\boldsymbol{\beta} \otimes \boldsymbol{\beta} .
\end{aligned}
$$

\section{Three-electron system}

Although the Kronecker product of spin matrices may not be the most practical way for the systematic treatment of much larger systems of particles (see, for example, [3] for some cases of many-electron atoms and molecules) it is nonetheless instructive for few electrons because of its conceptual simplicity. In the case of three electrons the spin operators are given by

$\hat{S}_{q}=\hat{s}_{q}(1) \otimes \hat{1}(2) \otimes \hat{1}(3)+\hat{1}(1) \otimes \hat{s}_{q}(2) \otimes \hat{1}(3)+\hat{1}(1) \otimes \hat{1}(2) \otimes \hat{s}_{q}(3)$.

Therefore, the problem reduces to the application of the Kronecker-product rule to each of the terms in the right-hand side and then adding the resulting expressions. For example, for the first term we should calculate $\mathbf{s}_{q} \otimes \mathbf{I} \otimes \mathbf{I}$. A straighforward calculation leads to

$$
\begin{aligned}
\mathbf{S}_{x}= & \frac{1}{2}\left(\begin{array}{cccccccc}
0 & 1 & 1 & 0 & 1 & 0 & 0 & 0 \\
1 & 0 & 0 & 1 & 0 & 1 & 0 & 0 \\
1 & 0 & 0 & 1 & 0 & 0 & 1 & 0 \\
0 & 1 & 1 & 0 & 0 & 0 & 0 & 1 \\
1 & 0 & 0 & 0 & 0 & 1 & 1 & 0 \\
0 & 1 & 0 & 0 & 1 & 0 & 0 & 1 \\
0 & 0 & 1 & 0 & 1 & 0 & 0 & 1 \\
0 & 0 & 0 & 1 & 0 & 1 & 1 & 0
\end{array}\right), \\
\mathbf{S}_{y}= & \frac{1}{2}\left(\begin{array}{cccccccc}
0 & -i & -i & 0 & -i & 0 & 0 & 0 \\
i & 0 & 0 & -i & 0 & -i & 0 & 0 \\
i & 0 & 0 & -i & 0 & 0 & -i & 0 \\
0 & i & i & 0 & 0 & 0 & 0 & -i \\
i & 0 & 0 & 0 & 0 & -i & -i & 0 \\
0 & i & 0 & 0 & i & 0 & 0 & -i \\
0 & 0 & i & 0 & i & 0 & 0 & -i \\
0 & 0 & 0 & i & 0 & i & i & 0
\end{array}\right),
\end{aligned}
$$




$$
\begin{aligned}
\mathbf{S}_{z}=\frac{1}{2}\left(\begin{array}{cccccccc}
3 & 0 & 0 & 0 & 0 & 0 & 0 & 0 \\
0 & 1 & 0 & 0 & 0 & 0 & 0 & 0 \\
0 & 0 & 1 & 0 & 0 & 0 & 0 & 0 \\
0 & 0 & 0 & -1 & 0 & 0 & 0 & 0 \\
0 & 0 & 0 & 0 & 1 & 0 & 0 & 0 \\
0 & 0 & 0 & 0 & 0 & -1 & 0 & 0 \\
0 & 0 & 0 & 0 & 0 & 0 & -1 & 0 \\
0 & 0 & 0 & 0 & 0 & 0 & 0 & -3
\end{array}\right), \\
\mathbf{S}^{2}=\frac{1}{4}\left(\begin{array}{cccccccc}
15 & 0 & 0 & 0 & 0 & 0 & 0 & 0 \\
0 & 7 & 1 & 0 & 1 & 0 & 0 & 0 \\
0 & 1 & 7 & 0 & 1 & 0 & 0 & 0 \\
0 & 0 & 0 & 7 & 0 & 1 & 1 & 0 \\
0 & 1 & 1 & 0 & 7 & 0 & 0 & 0 \\
0 & 0 & 0 & 1 & 0 & 7 & 1 & 0 \\
0 & 0 & 0 & 1 & 0 & 1 & 7 & 0 \\
0 & 0 & 0 & 0 & 0 & 0 & 0 & 15
\end{array}\right)
\end{aligned}
$$

As in the two-electron case the matrix $\mathbf{S}_{z}$ is diagonal but $\mathbf{S}^{2}$ is not; however, we can diagonalize the latter quite easily. The characteristic polynomial $\left|\mathbf{S}^{2}-\lambda \mathbf{I}\right|=4^{-8}(4 \lambda-3)^{4}$ $(4 \lambda-15)^{4}=0$, where $\mathbf{I}$ is the $8 \times 8$ identity matrix, shows that there are two eigenvalues: $\lambda=3 / 4(S=1 / 2)$ and $\lambda=15 / 4(S=3 / 2)$, each with four degenerate eigenvectors. The occurrence of four eigenvectors in the former case is due to the fact that the $\operatorname{spin} S=1 / 2$ appears twice. On the other hand, the four eigenvectors with spin $S=3 / 2$ come from the fact that $M_{S}=-3 / 2,-1 / 2,1 / 2,3 / 2$ as is well known. This result is also in agreement with the application of the abovementioned formula for the coupling of angular momenta to the coupling of the singlet $S=0$ and triplet $S=1$ two-electron spins with the spin $s=1 / 2$ of the remaining electron that yields $S=1 / 2$ in the former case and $S=1 / 2,3 / 2$ in the latter. The spin eigenvectors are

$$
\begin{aligned}
& |1 / 2,1 / 2\rangle=\frac{1}{\sqrt{2}}\left(\begin{array}{c}
0 \\
1 \\
-1 \\
0 \\
0 \\
0 \\
0 \\
0
\end{array}\right), \frac{1}{\sqrt{6}}\left(\begin{array}{c}
0 \\
0 \\
1 \\
0 \\
0 \\
0 \\
0 \\
0
\end{array}\right), \\
& |1 / 2,-1 / 2\rangle=\frac{1}{\sqrt{2}}\left(\begin{array}{c}
0 \\
0 \\
1 \\
0 \\
-1 \\
0 \\
0
\end{array}\right), \frac{1}{\sqrt{6}}\left(\begin{array}{c}
0 \\
1 \\
0 \\
1 \\
-2 \\
0
\end{array}\right),
\end{aligned}
$$




$$
\begin{aligned}
& |3 / 2,3 / 2\rangle=\left(\begin{array}{l}
1 \\
0 \\
0 \\
0 \\
0 \\
0 \\
0 \\
0
\end{array}\right), \quad|3 / 2,1 / 2\rangle=\frac{1}{\sqrt{3}}\left(\begin{array}{l}
0 \\
1 \\
1 \\
0 \\
1 \\
0 \\
0 \\
0
\end{array}\right), \\
& |3 / 2,-1 / 2\rangle=\frac{1}{\sqrt{3}}\left(\begin{array}{l}
0 \\
0 \\
0 \\
1 \\
0 \\
1 \\
1 \\
0
\end{array}\right),|3 / 2,-3 / 2\rangle=\left(\begin{array}{l}
0 \\
0 \\
0 \\
0 \\
0 \\
0 \\
0 \\
1
\end{array}\right) .
\end{aligned}
$$

The meaning of these eigenvectors is easy to understand if one simply compares them with all the Kronecker products of three one-electron spin eigenvectors; for example:

$$
\boldsymbol{\alpha} \otimes \boldsymbol{\alpha} \otimes \boldsymbol{\alpha}=\left(\begin{array}{l}
1 \\
0 \\
0 \\
0 \\
0 \\
0 \\
0 \\
0
\end{array}\right), \boldsymbol{\alpha} \otimes \boldsymbol{\alpha} \otimes \boldsymbol{\beta}=\left(\begin{array}{l}
0 \\
1 \\
0 \\
0 \\
0 \\
0 \\
0 \\
0
\end{array}\right), \quad \boldsymbol{\alpha} \otimes \boldsymbol{\beta} \otimes \boldsymbol{\beta}=\left(\begin{array}{l}
0 \\
0 \\
0 \\
1 \\
0 \\
0 \\
0 \\
0
\end{array}\right),
$$

etc.

\section{Two interacting spins}

As a final illustration of the utility of the Kronecker product we briefly discuss a simple model of two coupled spins given by the Hamiltonian

$$
\begin{aligned}
\hat{H}= & a \hat{s}_{z}(1) \otimes \hat{1}(2)+b \hat{1}(1) \otimes \hat{s}_{z}(2) \\
& +\lambda\left[\hat{s}_{x}(1) \otimes \hat{s}_{x}(2)+\hat{s}_{y}(1) \otimes \hat{s}_{y}(2)+\hat{s}_{z}(1) \otimes \hat{s}_{z}(2)\right] .
\end{aligned}
$$

This model is similar to the one used in the interpretation of the NMR signal produced by two protons (nuclear spin $I=1 / 2$ ) that experience two different local magnetic fields (the first two terms on the right-hand side) and are coupled by an interaction proportional to $\vec{s}(1) \cdot \vec{s}(2)$ (the third term on the right-hand side). The coupling term comes from the interaction of the magnetic momenta of the two nuclei that are proportional to the corresponding spin angular momenta and gives rise to the well known fine structure in the NMR spectrum [7]. 
In order to obtain the eigenvalues of this Hamiltonian we simply diagonalize the matrix

$$
\begin{aligned}
\mathbf{H}= & a \mathbf{s}_{z} \otimes \mathbf{I}+b \mathbf{I} \otimes \mathbf{s}_{z}+\lambda\left[\mathbf{s}_{x} \otimes \mathbf{s}_{x}+\mathbf{s}_{y} \otimes \mathbf{s}_{y}+\mathbf{s}_{z} \otimes \mathbf{s}_{z}\right] \\
& =\left(\begin{array}{llll}
\frac{a+b}{2}+\frac{\lambda}{4} & 0 & 0 & 0 \\
0 & \frac{a-b}{2}-\frac{\lambda}{4} & \frac{\lambda}{2} & 0 \\
0 & \frac{\lambda}{2} & \frac{b-a}{2}-\frac{\lambda}{4} & 0 \\
0 & 0 & 0 & -\frac{a+b}{2}+\frac{\lambda}{4}
\end{array}\right)
\end{aligned}
$$

where I stands for the $2 \times 2$ identity matrix. Note that the application of the Kroneckerproduct rule is considerably more straightforward than the explicit calculation of the matrix elements of the Hamiltonian $\hat{H}$ in the tensor-product spin basis set [7]. The eigenvalues and eigenvectors are

$$
\begin{aligned}
& E_{1}=-\frac{a+b}{2}+\frac{\lambda}{4}, E_{2}=-\frac{1}{2} \sqrt{(a-b)^{2}+\lambda^{2}}-\frac{\lambda}{4}, \\
& E_{3}=\frac{1}{2} \sqrt{(a-b)^{2}+\lambda^{2}}+\frac{\lambda}{4}, \quad E_{4}=\frac{a+b}{2}+\frac{\lambda}{4}
\end{aligned}
$$

and

$$
\mathbf{v}_{1}=\left(\begin{array}{l}
0 \\
0 \\
0 \\
1
\end{array}\right), \quad \mathbf{v}_{2}=\left(\begin{array}{c}
0 \\
\frac{1}{\sqrt{(u-v)^{2}+1}} \\
\frac{v-u}{\sqrt{(u-v)^{2}+1}} \\
0
\end{array}\right), \quad \mathbf{v}_{3}=\left(\begin{array}{c}
0 \\
\frac{1}{\sqrt{(u+v)^{2}+1}} \\
\frac{v+u}{\sqrt{(u+v)^{2}+1}} \\
0
\end{array}\right), \quad \mathbf{v}_{4}=\left(\begin{array}{l}
1 \\
0 \\
0 \\
0
\end{array}\right)
$$

respectively, where

$$
u=\frac{\sqrt{(a-b)^{2}+\lambda^{2}}}{\lambda}, v=\frac{b-a}{\lambda} .
$$

Note that $\mathbf{v}_{1}=\boldsymbol{\beta} \otimes \boldsymbol{\beta}$ and $\mathbf{v}_{4}=\boldsymbol{\alpha} \otimes \boldsymbol{\alpha}$ while $\mathbf{v}_{2}$ and $\mathbf{v}_{3}$ are linear combinations of $\boldsymbol{\alpha} \otimes \boldsymbol{\beta}$ and $\boldsymbol{\beta} \otimes \boldsymbol{\alpha}$. The energy differences $E_{4}-E_{3}, E_{4}-E_{2}, E_{3}-E_{1}$ and $E_{2}-E_{1}$ yield the frequencies of the two doublets in the NMR signal. These are the only transitions allowed by a well known selection rule [7]. Within the notation chosen for the present model the transition probability between the states $\mathbf{v}_{i}$ and $\mathbf{v}_{j}$ is proportional to $\left|\mathbf{v}_{i}^{T} \cdot \mathbf{S}_{x} \cdot \mathbf{v}_{j}\right|^{2}$, where $T$ stands for transpose. One can verify that $\mathbf{v}_{1} \longleftrightarrow \mathbf{v}_{4}$ and $\mathbf{v}_{2} \longleftrightarrow \mathbf{v}_{3}$ are forbidden.

If $|a-b| \gg|\lambda|$ we can apply perturbation theory to first order and obtain

$$
E_{2} \approx-\frac{|a-b|}{2}-\frac{\lambda}{4}, E_{3} \approx \frac{|a-b|}{2}+\frac{\lambda}{4} .
$$

This approach is equivalent to just keeping the diagonal terms in the Hamiltonian matrix $\mathbf{H}$ and the linear combinations of direct products of spin vectors just mentioned reduce to the simple tensor products $\boldsymbol{\alpha} \otimes \boldsymbol{\beta}$ and $\boldsymbol{\beta} \otimes \boldsymbol{\alpha}$ (eigenvectors of order zero). Thus, the NMR transitions mentioned above are approximately given by $\boldsymbol{\alpha} \otimes \boldsymbol{\alpha} \longleftrightarrow \boldsymbol{\alpha} \otimes \boldsymbol{\beta}$, 
$\boldsymbol{\alpha} \otimes \boldsymbol{\alpha} \longleftrightarrow \beta \otimes \boldsymbol{\alpha}, \boldsymbol{\beta} \otimes \boldsymbol{\beta} \longleftrightarrow \alpha \otimes \boldsymbol{\beta}$ and $\boldsymbol{\beta} \otimes \boldsymbol{\beta} \longleftrightarrow \beta \otimes \boldsymbol{\alpha}$; in other words, only one spin 'flips' in a transition [7].

\section{Further comments and conclusions}

One of the purposes of this paper is to show that in quantum mechanics it is customary to use two different operator products. One of them is the composition of two operators defined on the same vector space and the resulting operator is also defined on the same vector space. This product gives rise to the commutators $[\hat{A}, \hat{B}]=\hat{A} \hat{B}-\hat{B} \hat{A}$ that appear in the discussion of compatible observables, uncertainty principle, etc [1-3]. The matrix representation of the operator product in a given basis set is the product of the matrix representations of the factor operators in the same basis set.

The other product is the direct or tensor product of two operators defined on different vector spaces. The resulting operator is defined on a new vector space that is the direct or tensor product of the vector spaces of the factor operators. This tensor product is necessary when we move from a one-particle system to a multiparticle one. The most important property in this case is that the matrix representation of the resulting tensor-product operator is given by the Kronecker product of the matrix representations of the factor operators. The basis set is the tensor product of the basis sets for the vector spaces associated to each of the factor operators. The construction of the matrix representation of the tensor-product operator is given by a simple formula suitable for an introductory course on quantum mechanics that the students may easily apply to a wide variety of problems. Throughout this paper we have discussed three simple examples of the application of the Kronecker product that clearly show how to solve some quantum-mechanical problems in a systematic and straightforward way. We have selected spin problems because they are naturally given in terms of finite matrices (finite basis sets) but the procedure can be also applied to the case of infinite basis sets provided that we restrict to a finite subspace where we define finite matrices. In this case the results would be approximate and will give rise to, for example, the well known RayleighRitz variational method [3]. Many other interesting applications of the Kronecker product are available elsewhere [4-6].

The application of the Kronecker-product rule is simple and can be easily carried out by hand. However, it presents a good opportunity to introduce some elements of computer programming in quantum-mechanical courses. We suggest resorting to one of the many available computer algebra systems that are extremely powerful and useful for pedagogical purposes. They enable the student to construct the Kronecker product either interactively or by means of a suitable program, and diagonalize the resulting matrix in exact analytical way if it is possible or numerically in more complex cases. In the present case we have used the computer algebra system Derive (https://education.ti.com/en/us/home) and one of the Kronecker-product programs contributed to the Derive User Group (http://www.austromath. at/dug/).

\section{References}

[1] Messiah A 1976 Quantum Mechanics (Amsterdam: North-Holland)

[2] Cohen-Tannoudji C, Diu B and Laloë F 1977 Quantum Mechanics (Paris: Hermann)

[3] Pilar F L 1968 Elementary Quantum Chemistry (New York: McGraw-Hill Book Company)

[4] Nielsen M A and Chuang I L 2000 Quantum Computation and Quantum Information (Cambridge: Cambridge University Press) 
[5] Benenti G, Casati G and Strini G 2004 Principles of Quantum Computation and Information, Volume I: Basic Concepts (Hackensack, NJ: World Scientific)

[6] Benenti G, Casati G and Strini G 2007 Principles of Quantum Computation and Information, Volume II: Basic Tools and Special Topics (Hackensack, NJ: World Scientific)

[7] Becker E D 2000 High-Resolution NMR. Theory and Chemical Applications (San Diego, CA: Academic) 